\title{
Redimensión de las ideas pedagógicas de Lorenzo Luzuriaga desde una visión educativa aplicada.
}

\author{
Mayte Bejarano Franco \\ U. de Castilla La Mancha (España) \\ MariaTeresa.Bejarano@uclm.es \\ Javier RODRÍGUEZ TORRES \\ U. de Castilla La Mancha (España). \\ javier.rtorres@uclm.es
}

\section{Resumen}

Hablar actualmente de la figura pedagógica de Lorenzo Luzuriaga (1889-1959), significa ir más allá de las teorías, experiencias y principios educativos que propuso y del modelo organizativo que defendió (Luzuriaga, 1928, 1931, 1946). Implica, valorar un perfil multifacético que recoge tanto su faceta como pedagogo como la de editor, traductor, ideólogo, docente, tertuliano, padre, etc. en varios contextos: el español y el de cada uno de los países donde vivió y tuvo que exiliarse: Inglaterra y Argentina (19361959).

Sus ideales educativos quedaron plasmados en España a través de los distintos documentos como "Bases para un programa de instrucción pública" (1918). También mediante publicaciones y prácticas educativas. Tomemos como ejemplo de esto último, las Misiones Pedagógicas en las que participó (1931-1936). Desubicado de su patria, no dejó de trabajar sobre la idea de la Escuela Única en los distintos países en los que estuvo, pese a las difíciles circunstancias económicas que soportó.

En este artículo, presentamos la figura de Lorenzo Luzuriaga con una dimensión aplicada y práctica. Descubriremos la huella pedagógica que dejó en aquellas personas a las que dedicó gran parte de su vida en el último exilio argentino (1939-1959), con las que convivió, trabajó e instruyó en Tucumán y Buenos Aires (Argentina) y a las que aportó el firme ideal de que el progreso de un país, inevitablemente, ha de asentarse en la educación basada en la igualdad de oportunidades, en la justicia social y en el acceso a la cultura de toda la población.

Las principales aportaciones aquí presentadas son las entrevistas a modo de narraciones que hemos realizado a las alumnas y familiares del pedagogo, personas, algunas de ellas muy vinculadas a Luzuriaga y que nunca hasta ahora han sido entrevistadas. Sus opiniones y experiencias se recogieron durante en verano del año 2010 en Madrid (España), Tucumán y Buenos Aires (Argentina), y a través de ellas se desvela la enorme influencia ejercida por las ideas de pedagogo en prácticas institucionales y docentes que hoy día siguen estando vigentes.

Estas entrevistas narrativas forman parte de un amplio proyecto de investigación: Registros didácticos: itinerarios y trazas socio históricas en la enseñanza de la psicología en la formación docente. Las marcas en los profesores de psicología egresados de la UBA (1960 - 1976). Siendo un instrumento relevante, ya que las informantes ponen énfasis en las estructuras temporales, sociales y de significado muy reclamadas en este tipo de estudios (Kvale, 2011). En cada una de las narraciones se entregó una historia biográfica sustentada por vivencias desde la doble dimensión; personal y profesional. A través de ellas, obtuvimos aproximaciones cognitivas y lingüísticas sobre la importante figura del pedagogo.

Palabras claves: Luzuriaga, práctica educativa, narraciones, huella didáctica. 


\title{
Redimension of Lorenzo Luzuriaga's pedagogic ideas from an educational applied vision
}

\begin{abstract}
To speak nowadays about the pedagogic figure of Lorenzo Luzuriaga (1889-1959), it means to go beyond the theories, experiences and educational principles proposed by him and the organizational model which he defended (Luzuriaga 1928, 1931, 1946).It implies valuing a many- sided profile that contains his facets as a pedagogue as well as these of publisher, translator, ideologist, teacher, tertullian, father, etc. in several contexts: the Spanish one and those from each of the countries where he lived and had to go into exile: England and Argentina (1936- 1959).

His educational ideals were embodied in Spain through different documents as: "Basis for a public instruction program" (1918), publications and educational practices. Let's take as an example of the above mentioned, the Pedagogic Missions in which he took part in (1931-1936). Out of his mother land, he did not stop working on the idea of the Only School in the different countries where he stayed at, in spite of the difficult economic circumstances that he had to deal with.

In this article Lorenzo Luzuriaga's figure is presented with an applied and practical dimension. We will discover the pedagogic mark that he left in those people to whom he dedicated a great part of his life in his last Argentine exile (1939-1959), with them he coexisted, worked and those who he instructed in Tucuman and Buenos Aires (Argentina) providing them the firm ideal that the progress of a country, inevitably, has to settle itself in the education based on the equality of opportunities, on the social justice and on the access to culture of the whole population.

The main contribution of the work is his methodology, that is to say, the interviews as a kind of stories that we have realized to the pedagogue's pupils and relatives, people, some of them very linked to Luzuriaga and who they have never been interviewed till now. His opinions and experiences were gathered during the summer of the year 2010 in Madrid (Spain), Tucuman and Buenos Aires (Argentina) and through them is revealed the enormous influence exercised by the pedagogue's ideas in institutional and educational practices that continue being in force nowadays.

These narrative interviews are part of a wide project of investigation: Didactic Records: itineraries and socio- historical signs in the teaching of the psychology in the educational formation. The influences in the teachers of psychology graduated from the UBA (1960-1976). Being a relevant instrument, since the informants put emphasis in the temporary, social and meaning structures very claimed in this type of studies (Kvale, 2011). In each of the stories a biographical history sustained by experiences was delivered from the double dimension; personal and professional. Across them, we obtained cognitive and linguistic approximations on the important figure of the pedagogue.
\end{abstract}

Key words: Luzuriaga, educational practice, stories, didactic mark.

\section{Referencia normalizada:}

Bejarano Franco, M. y Rodríguez Torres, J. (2013) Redimensión de las ideas pedagógicas de Lorenzo Luzuriaga desde una visión educativa aplicada. Historia y Comunicación Social. Vol. 18. $\mathrm{N}^{\mathrm{o}}$ Especial Diciembre. Págs. 489-501.

Sumario: 1. Introducción. Estado de la cuestión. 2. La figura de Lorenzo Luzuriaga en la actualidad, un pedagogo redimensionado. 3. La dimensión aplicada de las ideas pedagógicas en Lorenzo Luzuriaga a través de la historia oral. 4. Análisis de los discursos. 5. Conclusiones. 6. Referencias Bibliografía

\section{Introducción. Estado de la cuestión.}

La situación educativa en España a finales del s XIX y comienzos del s. XX, se caracteriza por altos índices de analfabetismo, así se recoge en una de las obras de 
Cossío (1915) [1] quien indicaba a partir de un censo escolar de principio de siglo, la presencia de cuatro millones de niños, de los que 2,5 estaban sin escolarizar. Además los datos oficiales de la época, indicaban un $60 \%$ de analfabetismo en una sociedad agrícola y políticamente centrada en potenciar los derechos de las clases más acomodadas. Los liberales del s. XIX dejaron en un segundo plano la educación en el diseño de un modelo nacional progresista. El resultado de este modelo será muy gravoso para la mayoría de la población al cambio de siglo: un país analfabeto, con frecuente tendencia al enfrentamiento fratricida y una Iglesia Católica vigilante con afán inquisitorial. Se impedirá, por otra parte, la libertad de prensa e imprenta. En este contexto, el espacio para la escuela pública quedará muy reducido.

La Universidad a comienzos de siglo, también refuerza los privilegios de unos pocos, facilitando la promoción directa y casi exclusiva a puestos de ejercicio del poder. El profesorado universitario $<<$ privilegiado $>>$ utiliza su cátedra como plataforma política, desestimando cuestiones sociales y pedagógicas; como consecuencia la docencia es totalmente rutinaria y los métodos considerados más innovadores se centran en la magistralidad y la memoria.

No todos los intelectuales aceptarán la situación social y educativa. De entre ellos, surge la figura de Julián Sanz del Río (1814-1869) defensor del racionalismo armónico y de lo que se llamará Krausismo. Inspirándose en Krause diseñó un modelo ideológico (racionalismo armónico) de gran influencia en España a finales del s. XIX. Apostó por la doctrina laicista, que propugnaba la libertad de conciencia. Él junto a profesores de la Universidad, expulsados de sus cátedras, y atacados en el ejercicio de las libertades académicas, crearán en el año 1876 la Institución Libre de Enseñanza (ILE) consagrada al cultivo y propagación de la ciencia, la igualdad de oportunidades educativas para toda la población y el respeto ideológico. [2]

La Institución Libre de Enseñanza es completamente ajena a todo espíritu e interés de comunión religiosa, escuela filosófica o partido político; proclamando tan solo el principio de la libertad e inviolabilidad de la ciencia, y de la consiguiente independencia de su indagación y exposición respecto de cualquiera otra autoridad que la de la propia conciencia del Profesor, único responsable de sus doctrinas. (Art. 15 de los estatutos de la ILE)

De esta forma, la Institución Libre de Enseñanza nace entre un grupo de Catedráticos (Francisco Giner de los Ríos, Gumersindo de Azcárate y Nicolás Salmerón), separados de la Universidad por defender la libertad de cátedra y negarse a ajustar sus enseñanzas a los dogmas oficiales en materia religiosa, política o moral.

Si Giner de los Ríos (1939-1915) es el precursor de lo que será más tarde la Educación Nueva, Cossío (1892- 1977), será uno de los precursores de dos ideas claves en este comienzo de siglo la Escuela Activa y la Escuela Unificada, más tarde este último ideal lo trabajará intensamente Luzuriga (1889-1959) apostando por un modelo educativo centrado en la unificación del sistema educativo. Lorenzo se apoya en Giner y Cossío, y en los principios educativos institucionalistas a los que unió su propia historia académica. Estudió intensamente el estilo culturalista de Dilhey 
(1833-1911), Dewey (1859-1952), Decroly (1871-1932) y Montessori (1879-1952). Lorenzo, trabaja el concepto $<<$ Escuela Única $>>$, por la influencia que obtuvo de una de las estancias profesionales que llevó a cabo en Alemania (1909) que ensalzaba el pensamiento pedagógico europeo moderno. En 1912 en el BILE [3] ya se observan sus inquietudes, impaciencias y aproximaciones al término y concepto. Einheitschule que aparece en 1886 en Alemania con el significado de $<<$ Escuela Unificada $>>$ en respuesta a la simplificación y facilitamiento de oportunidades para todas las clases sociales en el acceso a estudios secundarios, además este movimiento pedagógico persigue los siguientes principios:

a) Establecer un tronco común para todas las enseñanzas.

b) Supresión de escuelas públicas para niños pertenecientes a diferentes clases sociales y establecer escuela básica y única para todos.

c) Se reduce el número de escuelas privadas para la preparación de la segunda enseñanza y por tanto, se acentúa el carácter público de este tramo educativo.

En definitiva, se propugnaba la eliminación de discriminaciones por razones económicas, sociales, sexuales, de religión, de raza, de ideología... para la educación de los ciudadanos y se hacía evidente la reivindicación del principio de igualdad de oportunidades como soporte del modelo educativo ahora propuesto.

Lorenzo, asume los principios inspiradores de la Escuela Unificada, así lo recoge en una de sus obras dedicada a este modelo (1931) y se convierte en uno de sus ardientes defensores, argumentando y aplicando soluciones a la situación particular de España en materia educativa. Acabará hablando de Escuela Única (1931), fruto de su célebre cuatrimembración pedagógica.
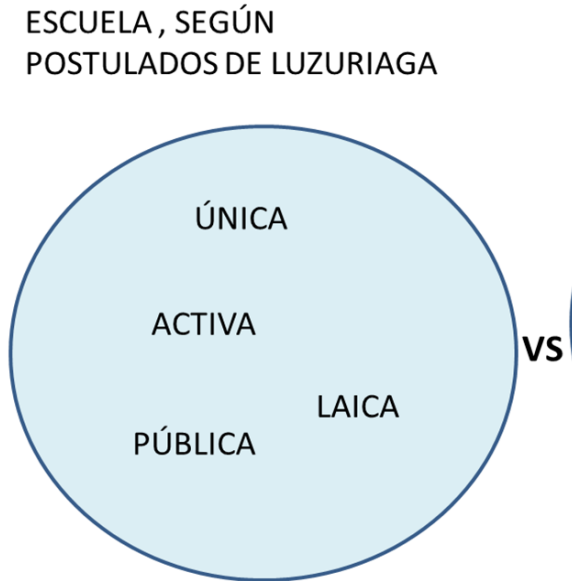

\section{ESCUELA, SEGÚN LA}

TRADICIÓN ESPAÑOLA

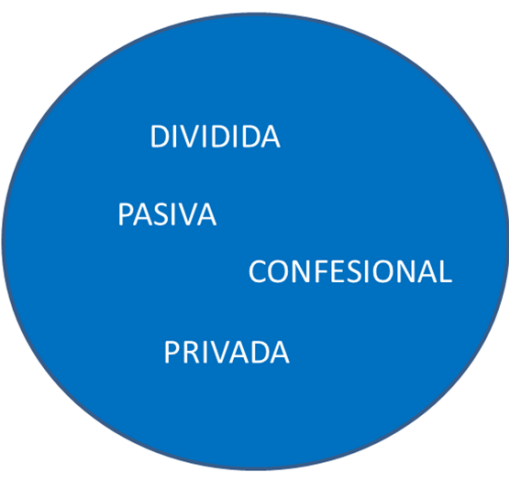

Figura 1: cuatrimembración pedagógica

Esta cuatrimembración cristaliza en su obra "La Nueva Escuela Pública” (1931), cuyos principios son parte importante de la aportación teórica - ideológica de la 
Ley Educativa Republicana, Ley de Instrucción Pública, encargada a Luzuriaga por Miguel de Unamuno y nunca aprobada. Sin embargo, algunos de esos planteamientos se hicieron realidad en un brevísimo espacio de tiempo, en el proyecto educativo progresista que el propio Lorenzo Luzuriaga impulsará desde la Secretaría del Ministerio de Instrucción Pública en el Primer Gobierno Republicano.

La Escuela Única tiene un profundo sentido de renovación política, técnica, metodológica y de instrumentos al servicio de los educadores. Lorenzo impulsa una renovación tanto externa como interna para el sistema educativo español y basándose en los principios de la Escuela Nueva llega a proponer gratuidad de la enseñanza y del material escolar para todos, pide asegurar el cubrir necesidades básicas como la comida y el vestir para los niños y niñas de familias no pudientes, mayor y mejor preparación para los maestros, así como sueldos unitarios para todo el personal docente (1931).

\section{La figura de Lorenzo Luzuriaga en la actualidad, un pedagogo redimensio- nado.}

Actualmente hablar de la figura pedagógica de Lorenzo Luzuriaga (1889-1959), significa ir más allá de las teorías, experiencias y principios educativos que propuso y del modelo organizativo que defendió (Luzuriaga, 1918, 1928, 1931, 1946). Significa, valorar un perfil multifacético que recoge tanto al pedagogo como al editor, traductor, ideólogo, docente, tertuliano y padre. Ello en varios contextos: el español y el de cada uno de los países donde vivió exiliado: Inglaterra y Argentina (1936-1959).

Sus ideales educativos, algunos previamente explicados, quedaron plasmados en España a través de los distintos documentos como "Bases para un programa de instrucción pública" (1918). Del análisis de este documento se desprenden propuestas como: la importancia de un plan educativo que implicaba la supresión separatista entre enseñanza primaria y secundaria o la prolongación en la obligatoriedad de la enseñanza hasta los dieciocho años.

Desubicado de su patria, debido a los exilios que sufrió, no dejó de trabajar sobre la idea de la Escuela Unificada en los distintos países en los que estuvo, pese a las difíciles circunstancias vitales por las que tuvo que pasar.

Recuperamos y presentamos la figura de Lorenzo Luzuriaga desde una dimensión aplicada y para ello, además de haber revisado algunas de sus ideas pedagógicas, aportamos y visibilizamos la huella pedagógica con dimensión práctica que dejó en aquellas personas a las que dedicó gran parte de su vida en el último exilio argentino (1939-1959), personas con las que convivió, trabajó y a las que instruyó en la Universidad de Tucumán y Buenos Aires (Argentina), transmitiéndoles el firme ideal de que el progreso de un país, inevitablemente, ha de asentarse en la educación basada en la igualdad de oportunidades, en la justicia social y en el acceso a la cultura de toda la población. 


\section{La dimensión aplicada de las ideas pedagógicas en Lorenzo Luzuriaga. La historia oral como fuente de recogida de información.}

La historia oral ha estado presente en el repertorio que ha acompañado a las prácticas de extracción de datos en la investigación social. Utilizar la historia oral y las fuentes documentales que la acompañan (manuscritos, artículos de prensa, fotografías...) supone tener en cuenta el trabajo de investigación historiográfico remarcando un contexto determinado, atendiendo a formas de conocimiento y pensamiento que también son determinadas. Es una forma de analizar las modalidades de comunicación de masas, pero también de recomponer los hechos y las vivencias individuales que forman parte del legado personal con dimensión colectiva. Este legado, en muchas ocasiones, se adquiere por la interacción entre personas y por la relación que se establece con las instituciones.

Trabajar temas históricos desde y con fuentes orales, nos posibilita acceder a saberes adquiridos que son íntimos, que no están publicados y sin embargo en su aplicación profesional y dimensión social jugaron un papel importante. Presentamos en este texto parte de los discursos que subyacen en las entrevistas realizadas a alumnas y colaboradoras que Lorenzo Luzuriaga tuvo en su exilio argentino, - también de su hija - y que formaron parte de una investigación iniciada en el año 2010 que tiene como principal sujeto de estudio la figura Lorenzo Luzuriaga desde el plano pedagógico y personal. Se contactó con dos de las alumnas en Tucumán (Argentina), destino latinoamericano de exilio donde ejerció docencia universitaria. En Buenos Aires entrevistamos a otra de sus discípulas y a su más cercana colaboradora en la Editorial Losada.

Lo que en principio se diseñó como entrevistas semi-estructuradas, se convirtió en auténticas narraciones que tuvieron como eje central la historia vital de cada informante debido a la huella pedagógica y personal dejada por el pedagogo. La intención de acceder y analizar el discurso pedagógico elaborado por estas discípulas, fue el registrar parte de la memoria colectiva educativa y contrastar si la formación obtenida en las clases universitarias llevadas a cabo por el pedagogo español tuvo continuidad en prácticas y estilos pedagógicos concretos. El registro oral supone una manera de complementar lo que otros profesionales escribieron sobre la obra del autor y de descubrir el impacto de su pensamiento. Entendemos por impacto, la concreción del discurso pedagógico en otros discursos construidos a través de la interacción pensamiento-práctica y que dan lugar a "otros planos históricos". Como afirma Fraser (1990) los testimonios cuentan no sólo el recuerdo sino un pensamiento y una actuación de época.

El análisis recogido de las narraciones, nos aproxima la marca educativa de las enseñanzas que Luzuriaga dejó en estas profesionales y que visibiliza un legado de conocimientos con diversidad de formas cognitivas vinculadas a la disciplina Pedagógica. Como mantiene Vilanova (1988) complementa la intención de capturar sentidos de la vida social y profesional y rompen el aislamiento y la soledad que en muchas ocasiones acompaña al mundo concluido de lo escrito. Otorga viveza y aporta sentido 
a las prácticas hoy vigentes que han sido trasladadas a lo largo de décadas hasta los espacios educativos. La narración de cada informante, se convirtió en un testimonio comunal (Bornat 2004; Yow 1994) debido a la trascendencia socio-educativa, que se reveló en cada una de las entrevistas. El legado $<<$ Luzuriaga $>>$, fue proyectado por estas alumnas en las instituciones en las que ejercieron como profesionales.

En estos discursos está la $<<$ llave $>>$ que intenta vincular pasado y futuro. Avisa a los que vienen detrás qué pasó antes. Con interpelaciones cuidadas se pueden encontrar respuestas narrativas, a veces compartidas y a veces discordantes. En su conjunto revisan proyecciones de experiencias que dejaron marca y que permiten que el vacío del tiempo o la ausencia puedan ser llenados.

La metodología de la historia oral repone y preserva informaciones en relación a diversos tópicos de la historia de la vida de las personas, de sus obras, del contexto de producción, de su proyección. También supone la posibilidad de tomar contacto con el hecho histórico a través de la evocación. La memoria, dejada en los discursos, supuso el compendio de recuerdos soportados por las emociones que se tradujeron en palabras. De esas palabras hilvanadas aparecieron discursos dejando como herencia educativa el conocimiento. Para quienes investigamos con esta metodología, supone el acceso a los hechos y a las realidades del pasado a través del presente y mediante una fuente de datos tangible y viva (Bejarano y Rodríguez, 2012).

\section{Análisis de los discursos.}

Estos discursos se convirtieron en un síntoma sobre la socialización educativa colectiva. A la vez no sólo constatábamos cómo Luzuriaga había trasladado en sus clases el modelo democratizador de la Escuela Única, sino que se recogía un saber que se hizo popular por a cuantas personas llegó y ello hizo que se convirtiera en un saber social redimensionado en los diferentes espacios educativos y de difusión editorial en los que Lorenzo Luzuriaga trabajo en el país argentino.

Recogemos en voz de sus discípulas y familiares la dimensión pedagógica, ideológica, educativa y personal que dejó la figura de Luzuriaga sin olvidar su condición de exiliado. Así nos lo revela su hija Isabel.

"No te puedes imaginar a la familia Luzuriaga corriendo, porque les van a meter a la cárcel, de país en país, de provincia en provincia porque hombre, iporque son rojos! Porque ¡fíjate qué ideas tienen sobre educación! (...) La familia Luzuriaga es exilio... lucha, hambre, salir corriendo de un momento para otro, nuevo exilio". (Luzuriaga, Isabel; testimonio oral; Madrid; 2010)

Lorenzo Luzuriaga, dinamizó ideológica, política y pedagógicamente espacios públicos y privados, primero en la España republicana y después en la pre-franquista. Fue un compromiso vital el que tuvo con la educación popular.

“El $80 \%$ de la gente era analfabeta, era lo que afligía a mi padre, porque él venía de un hogar donde no eran analfabetos pero eran gente modesta. De modo que él no quería que eso pasara. Persona de servicio que entraba en casa, persona que salía con una carrera. Eran cuatro: una que venía los lunes a lavar, la segunda que venía los 
martes a planchar (...) La otra que venía todos los días (...) otra que venía a cuidar a mi abuela, que estaba como estoy yo ahora" (Luzuriaga, Isabel; testimonio oral; Madrid; 2010)

Quizá este compromiso con la igualdad educativa constituyó una de las principales causas que le llevó a los exilios junto con su mujer y dos de sus hijos. Su hijo mayor, Jorge, permanece en España, encarcelado. Repasemos en voz de las informantes partes del recorrido vital y académico del pedagogo.

En 1936 sale exiliado para Londres, se traslada después a Glasgow, y allí trabaja impartiendo cursos de español. Pero nunca abandonó la producción para difundir sus ideales vinculados a la Escuela Única. Después viaja con toda su familia a Tucumán. Ejerció como profesor de Pedagogía en la Universidad Nacional de esta ciudad (1939). Años más tarde (1944), fue nombrado Vicedecano de la Facultad de Filosofía y Letras de dicha Universidad. Allí, en Tucumán, es reconocido por su implicación con la educación y su particular forma de impartir clases.

\footnotetext{
"Era parte de aquel conjunto de "maestros", auténticos, que llegaron para enseñar y formar; dispuestos a transmitir el saber y compartir con sus alumnos, sus valiosas y personales experiencias de vida, dentro y fuera de las aulas, [...]. [...] Aquella acción educadora dejó huellas indelebles, marcadoras de rumbos, en muchas trayectorias juveniles, pero significó fundamentalmente un impulso de incuestionable valor para la cultura $[\ldots] "$

“Allí estaba Don Lorenzo, como siempre: sereno, parco, sencillo, generoso, el que sin poses ni estridencias me entregó el conocimiento que él poseía- mucho y profundo- y su sabiduría, traducida en aquel consejo orientador y estimulante que solo los "grandes" pueden ofrecer y que tanto necesitaba yo para continuar, segura, con mi tarea educativa" (Dappe de Cuenya, María Elena; testimonio oral; Tucumán; 2010)
}

Con la condición de exiliado republicano dejó su pensamiento educativo plasmado en la amplia obra que fue produciendo en cada exilio y con la que hoy contamos a ambos lados del Atlántico. La obra, se identifica en libros, diccionarios, y artículos de revistas. Esta obra, da cuenta de que sus ideas pedagógicas fueron renovadoras y que no sólo se centraron en principios pedagógicos aplicados al "arte de enseñar", también estuvieron dirigidas a la formación práctica de pedagogos y maestras noveles que se instruían, primero, en espacios españoles y después en los argentinos.

Hoy, interesa reconocer sus ideas redimensionadas pues siguen vigentes, vivas y actuales. Se identifican en documentos, pero sobre todo en los testimonios de quienes asistieron a sus clases universitarias entregando sabiduría práctica.

Acercando la palabra de las discípulas y familiares del pedagogo, aproximamos un pedazo de la historia de la Pedagogía Moderna. Los discursos recogidos están guiados por los principios de la democratización de la enseñanza y la Escuela Única. Dan cuenta de la magnitud e importancia que han tenido los postulados pedagógicos de Luzuriaga, centrados en: la educación popular y pública, la unificación de etapas 
educativas, la importancia de la formación de los maestros y el inmenso interés por el niño como centro de la enseñanza-aprendizaje y la extinción del analfabetismo.

"Él ya en España demostró mucha simpatía y adhesión al socialismo y su tarea en la educación pública estuvo vinculada a la República en cuanto se instauró la República, hubo un movimiento de transformación de la enseñanza española muy importante, fue uno de los objetivos de la República. Él trabajó para mejorar la calidad educativa, combatir el analfabetismo, por la formación de maestros; en todo eso intervino Luzuriaga muy activamente a través de la Revista de Pedagogía que la había fundado en el año '22" (Cruzalegui, Laura; testimonio oral; Los Martínez, Buenos Aires; 2010

Los testimonios revelan cuáles fueron los condicionamientos y proyectos que orientaron su producción pedagógica en relación a: la docencia, la creación académica y la aplicación práctica de la ideología educativa. Todo esto quedó patente en las instituciones educativas por las que pasó, impulsó, fundó y las que luego retomaron su legado. Entre estas instituciones destacamos la Escuela Vocacional de Sarmiento de Educación Secundaria, en Tucumán, a la que se acudía también para realizar prácticas sobre la carrera del Magisterio. Allí también llegaron las influencias de las ideas de Lorenzo Luzuriaga, pues acudía con frecuencia a esta institución. Lucía Piosev Previch, alumna suya, nos deja el siguiente testimonio:

"La escuela Vocacional Sarmiento, por supuesto... como le digo, las ideas de la Escuela Nueva de Lorenzo Luzuriaga cayeron en un terreno pedagógico abonado por Clotilde Alonso Donate quien fue directiva de esta Escuela y estudiosa de esta corriente pedagógica. Comenzó su labor suprimiendo los bancos tradicionales, reemplazándolos por mesitas individuales, podíamos llevar las mesas al patio para realizar nuestras tareas. Fue una experiencia muy linda"

"Era una enseñanza que apelaba mucho a la libertad, pero al mismo tiempo nos creaba un sentimiento de responsabilidad muy grande, podíamos hacer..., para enseñarnos se utilizaban experimentos con elementos cotidianos, podíamos llevar animales al aula" (Lucía Piosev Previch, testimonio oral, Hierbabuena, Tucumán; 2010)

En este mismo sentido se pronunciaba la hija de Lorenzo Luzuriaga al ser preguntada por los principios pedagógicos de esta escuela Vocacional en la cual se formó:

"Eran clases activas. Te daban un programa de lo que ese mes ibas a estudiar y entonces te decían que si querías estudiar dentro de la clase o hacerlo por los jardines, y hacerlo como tú quisieras, es decir si no te apetecía hacer el programa de matemáticas pues acababas el de letras. Me auto-regulaba el aprendizaje. Cumplíamos con el programa en el mes pero como cada cual quería" (Luzuriaga, Isabel; testimonio oral; Madrid; 2010)

Aludimos ahora, al Jardín de Infancia Platero. Otra de las instituciones educativas que empezó su andadura bajo la influencia de las ideas educativas del pedagogo. Ubicado en Buenos Aires, llegó a contar hasta con dos sedes. Impulsado y creado desde ideas pedagógicas basadas en la Escuela Activa contó entre sus fundadoras con Isabel Luzuriaga - su hija - y la alumna Laura Cruzalegui. Esta última, revelaba la importancia de la actividad al aire libre, del ejercicio físico, de los talleres de creati- 
vidad en Platero. Estos principios pedagógicos básicos ya fueron propugnados en la República Española España.

"Nos parecía estimulante... la idea de la auto-valía del chico... esto lo fomentábamos mucho. Que los chicos se sintieran seguros. La primera infancia se consideraba básica, hacíamos mucha ejercitación física. Creamos la red mediante la cual los chicos saltaban y fue recogida en revistas americanas. Era un Jardín con muchos elementos que los chicos podían manejar libremente, aunque a veces dirigidos: el juego libre espontáneo para nosotras tenía mucha importancia" (Cruzalegui, Laura; testimonio oral; Los Martínez (Buenos Aires, 2010)

"El Jardín funcionó con los principios pedagógicos de mi padre Lorenzo Luzuriaga y de mi madre $\mathrm{M}^{\mathrm{a}}$ Luisa Navarro, basado en la Escuela Nueva y en la Escuela Activa" (Luzuriaga, Isabel; testimonio oral; Madrid; 2010)

Se ocupó de difundir éstas y otras ideas educativas a través de las Cátedras de Historia de la Educación y de Pedagogía en la Universidad de Buenos Aires.

"Bueno... ante todo su creencia en la importancia de la educación a través de la escuela y de otras instituciones. Él consideraba que era un elemento un factor fundamental para el desarrollo social a través de la escuela. Bueno, después si, tenía un fervor digamos por la educación, también, ¿no? Porque la escuela fuera una institución para promover el desarrollo infantil, la buena convivencia con los maestros y los chicos... esto unido a un espíritu democrático; en él era fundamental" (Cruzalegui, Laura, testimonio oral; Buenos Aires; 2010)

Durante su estancia en Buenos Aires, empezó a trabajar como director de la Colección Pedagógica de la editorial Losada, cuyo comité de lectura lo constituía un nidal de refugiados republicanos españoles. La presencia de éstos intelectuales en Losada enriqueció la producción de la editorial y también la cultura del país. Así lo señalaba su colaboradora en Losada.

"Luzuriaga impulsó a la Editorial. Era director de una colección. Todos estos intelectuales españoles contribuyeron mucho en la Editorial y en la cultura del país en aquellos años. El país recibió luminosidad intelectual en aquel momento [...] Lorenzo recorrió todo: la biblioteca clásica, la contemporánea, traducciones, prólogos, era tan brillante intelectualmente..." (Peremarti, Mabel, testimonio oral Buenos Aires; 2010)

Tradujo manuscritos de importante prestigio como Mi credo pedagógico y otras obras más de John Dewey, cuya filosofía pedagógica había contribuido a difundir en España desde 1916 junto con Domingo Barnés y Eugenio. También tradujo al castellano otras obras de Kirpatrick, Bühler y Wilhelm Messer, Dilthey, entre otros. No descuidó su propia producción: La pedagogía contemporánea (1942), Reforma de la educación (1945), Historia de la educación pública (1946), Pedagogía (1950), Pedagogía social y política (1954), Antología pedagógica (1956), La Institución Libre de Enseñanza y la educación en España (1957); ejerciendo una fuerte influencia sobre conocimientos y prácticas pedagógicas y didácticas. También trabajó para la editorial La Lectura. 
Pese a los exilios, no abandona su actividad política que lleva a cabo junto con otros españoles con su misma condición. Una muestra de esto, la encontramos en los relatos de Laura Cruzalegui y de Mabel Peremarti, quienes coinciden en afirmar que Luzuriaga era un asiduo de las tertulias políticas llevadas a cabo en la Casa de la literata Victoria Ocampo.

“En junio de 1947, Victoria Ocampo invitó a Luzuriaga a una recepción dada en honor de Julián HYPERLINK por entonces presidente de la UNESCO y amigo de la directiva de la revista Sur (que dirigía la literata). Allí denunció el genocidio cultural llevado a cabo por la dictadura franquista y esta denuncia se la hizo a un alto cargo de la UNESCO" (Cruzalegui, Laura, testimonio oral; Buenos Aires; 2010)

Algunos de sus alumnos/as llegaron a ocupar puestos educativos de reconocido prestigio en Argentina. $\mathrm{M}^{\mathrm{a}}$ Eugenia Valentié, Gustavo Bravo Figueroa, Josefa Sastre, Lucía Piosev entre otras personas ocuparon cátedras universitarias en materias pedagógicas, esta última, Lucía, además fundó el Instituto de Historia y Pensamientos Argentinos. María Elena Dappe fue directora de la Escuela Vocacional Sarmiento...

\section{Conclusiones.}

Apostar por la historia oral como fuente de recogida de información para esta investigación no solo aportó nuevos datos sobre la forma de vida académica y personal del pedagogo, supuso encontrar "las otras voces" de Lorenzo Luzuriaga. Si bien es cierto que el autor es conocido por las propuestas incluidas en el Movimiento de la Escuela Única, después de acercarnos a su historia vital a través de las narraciones, debemos preguntarnos si también es reconocido por "seguir haciendo pedagogía" en su condición de exiliado.

Los testimonios nos presentan una figura multifacética: maestro, profesor, pedagogo, político, escritor, editor. Su obra se materializa entre España y Argentina y se extiende más allá de fronteras territoriales y cronológicas, cuyos antecedentes aplicados se encuentran en la escuela republicana con marcas indelebles que se fusionan con experiencias y saberes de su exilio reformulando nuevas experiencias y saberes.

Nuestros objetivos al presentar este texto se basan en: tratar de identificar sus singulares trazas pedagógicas dejadas por su enseñanza en el aprendizaje de quienes le tuvieron como profesor; recorrer sus itinerarios académicos en voz de quienes le conocieron, recuperar legados acerca de la producción y del saber pedagógico en una época signada por cambios profundos guiados por los exilios.

Aun viviendo situaciones de partida e inicio provocadas por los exilios, lo tuvieron por protagonista y transmisor. Identificar interlocutoras de época y reponer experiencias educativas en las que se reconoce su marca pedagógica supone dar entidad y sentido a lo que sostiene un sistema educativo democrático y participativo. Es, ante 
todo hacer visible la materialización de una ideología sustentada por la igualdad y el valor que posee la educación.

"Aunque la tragedia de la guerra actual, con todos sus horrores y todos sus heroísmos, no haya llegado aún a su fin, parece necesario, si se quiere preparar un mundo mejor, una sociedad nueva, donde no sea posible su repetición, que se adopten desde ahora ciertas medidas (...) Si se aspira a una reforma o transformación del mundo de la postguerra, hay que empezar por cambiar al hombre actual y preparar al hombre de mañana de otro modo que se ha hecho hasta ahora. Y esta es función de la educación" (Luzuriaga, Lorenzo, 1945, Reforma de la Educación; Losada; Buenos Aires)

\section{Referencias y bibliografía}

COSSÍO M B (1915). La Enseñanza Primaria en España... Madrid 1915.

AZCÁRATE, P. (1967). "Notas sobre el origen de la Institución Libre de Enseñanza", Boletín de la Real Academia de la Historia, núm. 161, Madrid 1967.

Boletín Institución Libre de Enseñanza. Comenzó a publicarse cuatro meses después de la fundación de la Institución Libre de Enseñanza, el 7 de marzo de 1877. El BILE, además de informar sobre la marcha del centro, respondía a la constatada "necesidad de una publicación económica donde se dé a conocer el movimiento intelectual contemporáneo". Con este enfoque, pronto se convirtió en una publicación singular en el mundo editorial español por su carácter cosmopolita y multidisciplinar y por la gran calidad de sus colaboradores. Pedagogos, investigadores, filósofos y literatos

ALVÁREZ LÁZARO, P y UREÑA, E M. (coords) (1999). La actualidad del krausismo en su contexto europeo. Madrid: Coeditado por la Fundación Duques de Soria, la Editorial Parteluz y la Universidad Pontificia de Comillas.

ARANGUREN, J L. (1996). Moral y Sociedad. La moral social española en el siglo XIX, Madrid: Edicusa,

Bases para un programa de instrucción pública (1918). XI Congreso nacional del PSOE.

BEJARANO FRANCO, M. Y RODRÍGUEZ TORRES J. (2013). La recuperación de "las trazas didácticas de Lorenzo Luzuriaga" a través de la fuente oral en personas mayores. En Envejecimiento Activo y Solidaridad Intergeneracional. Madrid: UNED.

BORNAT, J. (2004). Oral history. En Seale, C. Gobo, G. Gubrium J.F y Silverman D (eds.), Qualitative Research Practice. Londres: Sage, pp. 34- 47.

FRASER R. (1990). La formación del entrevistador. En Historia y fuente oral, n 3 pp. 129-150.

KVALE, S. (2011). Las entrevistas en investigación cualitativa. Madrid: Morata.

LUZURIAGA, L. (1931). La Escuela Única. Madrid: Biblioteca Nueva.

LUZURIAGA, L. (1948) La escuela nueva pública. Buenos Aires: Losada.

VILANOVA, M (ed.). (1988). El poder en la sociedad. Barcelona: Antoni Bosch.

YOW, V. R. (1994). Recording Oral History. Thousand Oaks, CA: SAGE. 


\section{Los autores}

Mayte Bejarano Franco es Doctora en Ciencias de la Educación por la Universidad de Granada, profesora en la Facultad de Educación de Ciudad Real de la Universidad de Castilla - La Mancha. Sus líneas de investigación se centran en género - educación y nuevas metodologías de enseñanza, que se relacionan con distintos grupos de investigación, nacionales e internacionales, donde trabaja. Ha ocupado cargos de gestión en el Departamento de Pedagogía, al cual pertenece.

Javier Rodríguez Torres es Doctor en Pedagogía, Máster en Psicología Escolar, Licenciado en Pedagogía y Profesor de EGB. Durante más de veinte años, ha trabajado en la Enseñanza no universitaria. Desde el curso 2004, es Profesor en la Universidad de la UCLM en el Departamento de Pedagogía. En la actualidad, es Secretario del Departamento. Profesor de distintos cursos de Postgrado de la UCLM. Miembro de distintos Proyectos de Investigación con las Universidades de Alcalá, UCLM, UNED, Poitiers (Francia) y UBA. Sus líneas de investigación abordan el currículum en torno a las diferencias de género y la inclusión de las TIC, los discursos de poder -saber que se generan en el uso y aplicación de las tecnologías, educación en valores y convivencia y educación para salud. Ha obtenido premios nacionales, autonómicos y provinciales a distintos proyectos de investigación e innovación educativa. 